\title{
Stepwise Rehabilitation of the Triple Amputee Combined With Dysfunction of the Sound Limb
}

\author{
Ji Cheol Shin, MD, Hye Eun Yang, MD, Su Jin Yu, MD, Na Young Kim, MD, Seo Yeon Yoon, MD
}

Department of Rehabilitation Medicine and Research Institute of Rehabilitation Medicine, Yonsei University College of Medicine, Seoul, Korea

To find a multiple amputee more severe than a triple amputee is not easy. This is a report of a 36-year-old patient with right knee disarticulation, left trans-femoral amputation and right elbow disarticulation due to peripheral ischemic necrosis, when he was applied vasopressor in septic shock condition. His left hand was also 2nd, 3rd, 4th, and 5th distal interphalangeal joint disarticulation status, and it was more difficult for him to do rehabilitation program, such as donning and doffing the prostheses. For more efficient rehabilitation training program, we first focused on upper extremities function, since we believed that he might need a walking aid for gait training later. After 13 weeks of rehabilitation program, he has become sit to stand and walk short distance independently with an anterior walker. Although he still needs some assistance with activities of daily living, his Functional Independence Measure score improved from 48 to 90 during the course of 13 weeks.

Keywords Multiple limb amputation, Amputation rehabilitation, Hand contracture

\section{INTRODUCTION}

There have been few reports about a multiple amputee because such cases are very uncommon and successful rehabilitation program is very difficult for them [1-5]. Upper extremity amputation is relatively rare but when it does occur, it affects hand function. This is especially true when upper extremity and lower extremity amputations are combined. In such situations, the rehabilita-

Received October 25, 2012; Accepted May 14, 2013

Corresponding author: Seo Yeon Yoon

Department of Rehabilitation Medicine and Research Institute of Rehabilitation Medicine, Yonsei University College of Medicine, 50 Yonsei-ro, Seodaemun-gu, Seoul 120-749, Korea

Tel: +82-2-2227-2241, Fax: +82-2-364-0509, E-mail: danggn82@hanmail.net

(c) This is an open-access article distributed under the terms of the Creative Commons Attribution Non-Commercial License (http://creativecommons. org/licenses/by-nc/3.0) which permits unrestricted noncommercial use, distribution, and reproduction in any medium, provided the original work is properly cited.

Copyright (C) 2014 by Korean Academy of Rehabilitation Medicine tion program becomes more difficult since patients have the trouble of donning and doffing the prostheses by themselves. Thus, until now, an efficient rehabilitation program has not been organized [2,4]. Previous rehabilitation programs have failed because they only focused on the gait training. To overcome this failure, a stepwise approach rehabilitation program is needed. It is important not only to understand the patient's characteristics, such as age and occupation, but also set short-term and long-term goals. In addition, it is critical to define their priorities from initial stage of rehabilitation using a multidisciplinary team approach. In this paper, we report a successful rehabilitation program of a multiple amputee with right knee disarticulation, left trans-femoral amputation and right elbow disarticulation combined with hand disarticulation and contracture of sound left limb. Because this patient got not only both lower extremities amputation and right upper extremity amputation but also sound limb hand disarticulation and contracture, we 
first focused on sound limb hand function recovery before intensive gait training. We then trained him in activities of daily living (ADL) on wheelchair level to improve independency. After that, we measured prostheses and started gait training. This stepwise rehabilitation program enables a successful rehabilitation.

\section{CASE REPORT}

A 36-year-old man, who had splenectomy history due to trauma when he was 10 years old, visited the emergency room due to abdominal pain and vomiting on November 15th, 2010. He developed septic shock, so norepinephrine was applied for 5 days and peripheral ischemia of four extremities occurred. The patient underwent right knee disarticulation, left trans-femoral amputation and right elbow disarticulation on December 21th, 2010 (Fig. 1). He underwent skin flap operation of the right knee and left hand skin defect area on January 10th and 24th, 2011, and joint range of motion exercise and isometric exercise were performed. After the operation wound healing, he was admitted to the Severance Rehabilitation Hospital for prostheses measurement and rehabilitation on March 5th, 2011.

Considering the patient's young age, we set the final goals as gait, independency of ADL and going back

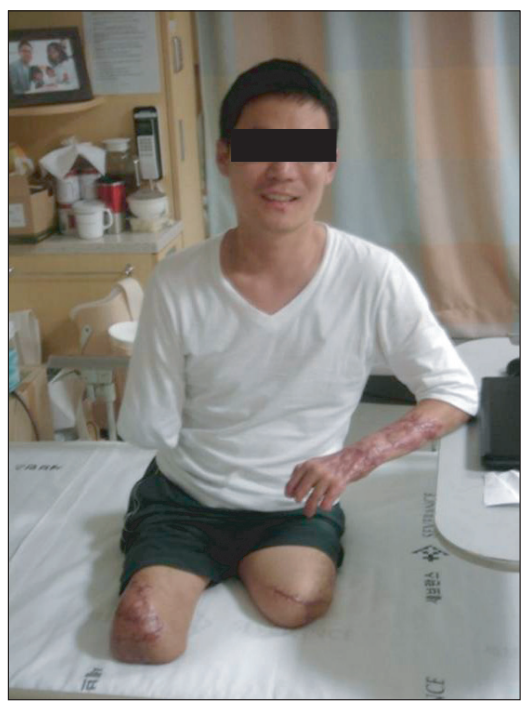

Fig. 1. Sitting without prosthesis (right knee disarticulation, left trans-femoral disarticulation, right elbow disarticulation, left 2nd, 3rd, 4th, and 5th distal interphalangeal joint disarticulation). to work. During the initial stage of the rehabilitation program, we focused on upper extremity function improvement and independency improvement on wheelchair level activity. After this short-term goal had been reached, gait training and car training were planned. Finally, before discharge, we planned to do computer training since he used computer a lot for his marketing business.

Before admittance to our hospital, the patient had a psychiatric consultation due to depression. There was no objective data for the depression score, but he showed clinically meaningful level of depression, so he took antidepressant medication. After symptoms improved, medication was stopped, and he no longer took the pills after being admitted to our hospital. Since his limb was amputated when he was on shock status, he finally recognized amputation after recovering from the shock. This made him feel negative body image of himself, and thus he went through anorexia and sociophobia. But, after admission to our hospital, he accepted his situation and got over with negative body image and wanted active rehabilitation. We helped him get actively involved in the rehabilitation program by inspiring a sense of accomplishment from stepwise functional recovery. Sometimes he felt the depression, but he did not want to take the medication. Continuous support from his family and inducing motivation made him feel better, so he could actively participate in the rehabilitation program. The patient's motivation was increased particularly by setting short-term goals that considered the patient's condition and by sharing the rehabilitation program with the patient. When he was admitted, the stump sites were relatively fine: right elbow disarticulation site was club shape and stump length from acromion was $32 \mathrm{~cm}$; right knee disarticulation site was cylindrical shape and stump length from great trochanter was $44.6 \mathrm{~cm}$; and left transfemoral amputation site was conical shape and stump length from great trochanter was $33.3 \mathrm{~cm}$. Skin condition and soft tissue shrinkage of all three amputation sites were relatively fine with no bony spurs or neuroma. There had been phantom pain and phantom sensation on stumps, but they decreased during gait training with the prostheses. Also, his left hand was 2nd, 3rd, 4th, and 5 th distal interphalangeal joint disarticulation status, skin flap site was fragile, and there were blisters due to friction during the rehabilitation program. This condition 
of the sound limb made the rehabilitation program more difficult for the patient. At first, passive range of motion of the left wrist joint flexion was $40^{\circ}$ and extension was $30^{\circ}$. In addition, 1st web space length was $2 \mathrm{~cm}$, but we could achieve $4 \mathrm{~cm}$ of 1st web space after left hand stretching exercise (Fig. 2). Due to the contracture and weakness of the left hand, he could not do opposition before intensive training, however, he was finally able to do tip pinch using thumb and 2nd finger when he picked up something like a tissue. Manual muscle power test (MMT) of upper extremities on admission showed grade 4 on both shoulder joints in terms of flexion, extension, abduction and adduction, grade 3 on the left elbow and wrist joint in terms of flexion and extension, and grade 2 on the left hand in terms of flexion and extension. MMT of lower extremities on admission showed grade 3 on both hip joints in terms of flexion, extension, external rotation and internal rotation. After strengthening exercise, the left hand muscle power improved to grade 3 , and the hip joint muscle power improved to grade 4 together with strengthening exercise and Cybex (Cybex 770+TMC) isokinetic training. Since he had stayed in the intensive care unit (ICU) for a long time, deconditioning and impaired pulmonary function also made his rehabilitation program more difficult. Vital capacity (VC) on admission was $3,480 \mathrm{~mL}$ (75.9\% of predictive value) and peak cough flow (PCF) on admission was $450 \mathrm{~mL}$. We guided him to buy an Ambu bag and trained him to do air stacking exercise, and finally, VC/PCF reached to 5,300 (115\%)/650.

During the 2nd week of the program, he was fitted with the right elbow disarticulation prosthesis. Elbow disarticulation socket, elbow flexion device control unit, elbow lock control cable, outside locking hinge, constant friction wrist unit, and figure of 8 harness were used. Artificial hand was used for cosmetic reasons during work, and bimanual activity training was performed. Even though he was originally right handed, we planned to make sound left hand as the dominant hand at first. His left hand function test was impossible due to the contracture and weakness on admission, but after training, pinch power showed $1.8 \mathrm{~kg}$ and Box and Block Test showed 33 points. However, there still remained some limitations to using the left hand as the dominant hand. First, the motion of the left wrist was limited as flexion $50^{\circ}$ extension $50^{\circ}$, and skin condition was still thin and fragile. Second, due to remaining contracture, it seemed hard to bear his weight with the left hand, even though the left hand function was recovered. So, we changed our plan and decided his right hand as the dominant hand and trained each hand the same proportionally. Before intensive gait training, we measured wheelchair (MIKI U2; MIKI Korea, Daegu, Korea), did ADL training with prosthesis on wheelchair level, and made him improve independency and use walking aid more efficiently after. Sitting balance was good on admission but ADL training had not been done; so Functional Independence Measure (FIM) score of self care subunit was 6 points. So intensive ADL training was done and he finally could don upper prosthesis by himself using pull over sweater method. But, since dressing, eating and grooming still needed minimal assistance due to the impaired hand fine motor function, ADL training was done consistently until discharge. On occupational therapy, ADL training, such as spoon use, drinking with a cup, and buttoning and unbuttoning, was done and as a result, follow-up FIM score improved to 12 after 1 month and 25 on discharge. He was able to do upper dressing by himself, although it took 2 minutes and 30 seconds. We decided on the dominant leg as the right leg because of the longer stump length on the right side. We also decided on the height of $162 \mathrm{~cm}$, which is shorter
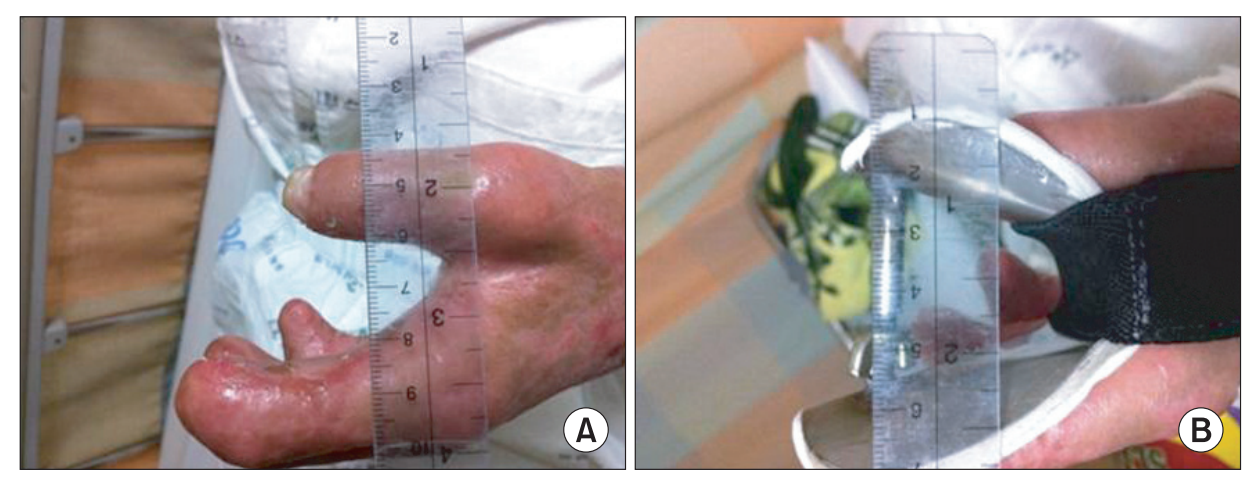

Fig. 2. Improvement of 1 st web space length: (A) $2 \mathrm{~cm}$, (B) $4 \mathrm{~cm}$. 
than his original height of $167 \mathrm{~cm}$, to improve stability by lowering the center of gravity. The patient was fitted with right knee disarticulation prosthesis with end bearing socket, polycentric knee, endoskeletal shank and dynamic solid ankle cushion heel (SACH) foot on the 6th week of the program. In addition, he was fitted with left transfemoral amputation prosthesis with quadrilateral socket, polycentric knee, endoskeletal shank and dynamic SACH foot on the 8th week of the program. Parallel bar weight shift training was started on the 9th week of the program. On the 10th week of the program, he started gait training with anterior walker without wheels, and he finally could walk $100 \mathrm{~m}$ using anterior walker without pause (Fig. 3). After starting intensive gait training, we analyzed the gait pattern and found that the left leg was dragged on swing phase. We shortened the left leg by a quarter inch and it improved the gait pattern. During rehabilitation program, doctor, physical therapist, occupational therapist, and prosthetist regularly met twice a week and discussed the prostheses fitting, hand function recovery, and gait pattern. This multidisciplinary team approach made the patient more motivated and rehabilitation program more efficient.

Cane gait seemed to be difficult for him due to the left hand contracture and weakness. We decided to gait with a walker indoors and use an electronic wheelchair outdoors. Family education, such as stump site management, was done and occupational rehabilitation pro-

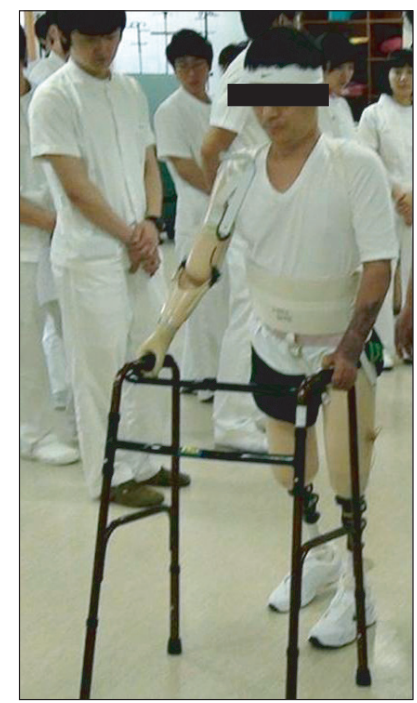

Fig. 3. Gait training with anterior walker after fitting prosthesises. gram, such as ADL training and computer training, was done for going back to work. We rented a car for disabled people and trained him to move to the driver's seat and drive short distance with electronic wheelchair. And we also gave him information about repair costs of a car for the disabled. He could use a computer mouse by himself, but since the typing speed was too slow, a universal cuff was used for faster speed. On discharge, ADL independency was increased up to 90 point of FIM score and 56 point of Modified Barthel Index score, which were 48 point and 20 point on admission, respectively (Table 1 ).

Functional level status also improved. He was at absolute bed rest status on admission, but at the time of discharge, he could come to sit by himself and gait with anterior walker. After discharge, we follow up on him constantly. He is successfully goes back to work using an electronic wheelchair and performs almost all of ADL by himself without help on wheelchair level.

Table 1. Improvement of activities of daily living

\begin{tabular}{|ccc}
\hline & $\begin{array}{c}\text { Admission } \\
\text { (Mar 7, 201 1) }\end{array}$ & $\begin{array}{c}\text { Discharge } \\
\text { (Jun 13, 2011) }\end{array}$ \\
\hline FIM & & \\
\hline Self-care & 6 & 25 \\
\hline Sphincter control & 2 & 14 \\
\hline Transfers & 3 & 10 \\
\hline Locomotion & 2 & 6 \\
\hline Communication & 14 & 14 \\
\hline Social cognition & 21 & 21 \\
\hline Total & 48 & 90 \\
\hline MBI & & \\
\hline Personal hygiene & 0 & 3 \\
\hline Bathing self & 0 & 1 \\
\hline Feeding & 0 & 5 \\
\hline Toilet & 0 & 2 \\
\hline Stair climbing & 0 & 0 \\
\hline Dressing & 0 & 5 \\
\hline $\begin{array}{l}\text { Bower \& bladder } \\
\text { control }\end{array}$ & 20 & 20 \\
\hline Ambulation or & & \\
\hline wheelchair & 0 & 12 \\
\hline Chair/bed transfer & 0 & 56 \\
\hline Total & 20 & \\
\hline F & & \\
\hline
\end{tabular}

FIM, Functional Independence Measure; MBI, Modified Barthel Index. 


\section{DISCUSSION}

Amputation rehabilitation is very complicated and it is difficult to make many decisions, including when to fit prosthesis, when to start gait training, and when to move on to the next step. It needs a comprehensive team approach. Rehabilitation for a multiple amputee is even more complicated and there are many considerable factors during the rehabilitation program. A triple amputee is very rare, and there have been few reports about successful rehabilitation of a multiple amputee more severe than a triple amputee [1-5]. Kitowski and Leavitt [1] reported independent gait training of a quadruple amputee with bilateral trans-radial and trans-femoral amputations using a systematic and multidisciplinary team approach. Shin et al. [2] reported quad-cane gait of a triple amputee with left trans-humeral amputation, right transtibial amputation, and left hip disarticulation.

Despite the limited number of reports, a triple amputee can usually walk with a cane by himself after fitting prostheses and gait training. Even though the triple amputee in our case had a sound left hand, it was hard to decide the dominant hand on the initial stage of rehabilitation program due to its interphalangeal joint disarticulation with contracture. We were not sure whether he would be able to use a cane with the left hand or not after the hand function recovery. Consequently, we had to focus on the left hand function recovery first and set the final goal after that. Generally, when deciding the dominant hand of bilateral upper extremities amputations, stump length, MMT, and range of motion are major considerable factors. Thus, we carefully compared the condition and recovery speed of both upper extremities to each other on that point. In this case, even though strengthening and stretching exercise of bilateral upper extremities were performed while measuring the right prosthesis, and the left hand function was improved up to tip pinch using thumb and 2nd finger, but skin of the left hand was so fragile and weak that it seemed impossible to use a cane with the left hand. After this, we changed our plan for the dominant hand from the left hand to the right hand. Therefore, for a rehabilitation program of a multiple amputee, both the short-term and long-term goals should be set properly, and stepwise approach is needed. If we cannot reach the short-term goal, we should modify the goals flexibly considering all circumstances of the pa- tient.

A successful rehabilitation program requires a number of important factors including a multidisciplinary team approach, information about the patient such as occupation, family and home circumstance, psychological support, fitting prosthesis at a proper time, and family education. Moreover, understanding disability from amputation, agreement on goal setting of doctors, and motivation about the rehabilitation program of a patient are also important. Shaw et al. [3] reported that not only proper prostheses fitting time but also psychosocial factors are important for the rehabilitation of a multiple amputee. This patient had depression, including anorexia and social phobia symptoms. He took a psychological consultation and medications before being admitted to our hospital. During the rehabilitation program at our hospital, we improved his motivation through sufficient explanation and sharing of short-term and longterm goals. Accomplishment of stepwise goals made him more actively involved in the program. Once amputation occurs, normal physiology is disrupted. Even if a patient can walk independently after the rehabilitation program, energy consumption and oxygen consumption will increase compared to a normal person. Though the amount of increase in energy and oxygen consumption varies somewhat depending on the authors, generally, trans-femoral amputation and transtibial amputation with prosthesis need $75 \%$ more energy consumption and bilateral trans-femoral amputations with prostheses need $110 \%$ more energy consumption compared to normal persons. Huang et al. [6] reported that bilateral transfemoral amputee walks $30 \%$ slowly and consumes $280 \%$ more energy compared to normal persons. Hoffman et al. [7] reported that trans-femoral amputee consumes $49 \%$ more oxygen compared to a normal person. These energy and oxygen consumption increases can be somewhat reduced through rehabilitation program. Park et al. [5] reported that a triple amputee, who showed $318 \%$ of oxygen consumption, improved to $285 \%$ after a rehabilitation program lasting 8 weeks. This implies that for efficient energy and oxygen consumption, rehabilitation program is crucial. The patient in our case had been in the ICU for a long time, so his general condition was poor and pulmonary function was decreased on admission. Rehabilitation was necessary to improve his pulmonary function for gait training and to protect reduced pul- 
monary function due to mobility restriction. As a result, we improved his pulmonary function on discharge. Although he will use an electronic wheelchair for outdoor activities, we recommend him to do gait training with an anterior walker at indoor level in order to maintain the muscle mass. A lot of cases have been reported about patients who are discharged to home and the society and who do not don the prostheses, leading to functional level decreases, and joint contracture development.

This kind of patient education is very important and meaningful so that the patients understand the physiological changes of their body, keep their body in optimal condition for lifetime, make them actively involved in the rehabilitation program, and make them feel satisfaction and a sense of accomplishment. Ultimately, we report this case as a successful rehabilitation of a triple amputee.

\section{CONFLICT OF INTEREST}

No potential conflict of interest relevant to this article was reported.

\section{REFERENCES}

1. Kitowski VJ, Leavitt LA. Rehabilitation of a quadruple amputee. South Med J 1968;61:912-4.

2. Shin JC, Park CI, Kim YC, Jang SH, Bang IK, Shin JS. Rehabilitation of a triple amputee including a hip disarticulation. Prosthet Orthot Int 1998;22:251-3.

3. Shaw M, Kaplow M, Mitchell N, Stillwell D. Traumatic triple amputation: psycho-social problems in rehabilitation. Arch Phys Med Rehabil 1977;58:460-2.

4. Davidson JH, Jones LE, Cornet J, Cittarelli T. Management of the multiple limb amputee. Disabil Rehabil 2002;24:688-99.

5. Park JM, Kim YJ, Kim IS, Lee YT, Kim SH, Kim YH, et al. Rehabilitation for ambulation of triple amputee: a case report. J Korean Acad Rehabil Med 2007;31:123-6.

6. Huang CT, Jackson JR, Moore NB, Fine PR, Kuhlemeier KV, Traugh GH, et al. Amputation: energy cost of ambulation. Arch Phys Med Rehabil 1979;60:18-24.

7. Hoffman MD, Sheldahl LM, Buley KJ, Sandford PR. Physiological comparison of walking among bilateral above-knee amputee and able-bodied subjects, and a model to account for the differences in metabolic cost. Arch Phys Med Rehabil 1997;78:385-92. 